\title{
Preparative separation of ketoprofen enantiomers: Choice of mobile phase composition and measurement of competitive adsorption isotherms
}

\author{
António E. Ribeiro ${ }^{a}$, Nuno S. Graça ${ }^{a}$, Luís S. Pais ${ }^{a}{ }^{a}$, Alírio E. Rodrigues ${ }^{b}$ \\ ${ }^{a}$ Laboratory of Separation and Reaction Engineering, School of Technology and Management, Bragança Polytechnic Institute, \\ Campus de Santa Apolónia, Apartado 1134, 5301-857 Bragança, Portugal \\ ${ }^{\mathrm{b}}$ Laboratory of Separation and Reaction Engineering, Faculty of Engineering, University of Porto, \\ Rua Dr. Roberto Frias s/ $n$, 4200-465 Porto, Portugal
}

Received 16 May 2007; received in revised form 9 November 2007; accepted 21 November 2007

\begin{abstract}
The present work intends to investigate how mobile phase composition influences the adsorption behavior of ketoprofen enantiomers (a nonsteroidal anti-inflammatory drug) on an amylose-based chiral stationary phase (Chiralpak AD). Three mobile phase compositions were studied: the usual $20 \%$ ethanol/80\% $n$-hexane mixture and two pure mobile phases; methanol and ethanol. Pulse and breakthrough experiments under preparative conditions were carried out in order to measure and test adsorption isotherms. The results obtained show that, for preparative separations, pure ethanol is a better mobile phase than the usual 20\% ethanol/80\% $n$-hexane mixture: it allows higher solubility of the racemate, lower retention times, and also a higher selectivity at high enantiomer concentrations. These are aspects of crucial importance when the final goal is to achieve high productivity preparative separations, as it is shown for the simulated moving bed (SMB) operation.
\end{abstract}

(C) 2007 Elsevier B.V. All rights reserved.

Keywords: Ketoprofen; Enantiomer separation; Mobile phase composition; Preparative chromatography; Simulated moving bed

\section{Introduction}

Ketoprofen $(R, S) 2$-(3-benzoylphenylpropionic acid) enantiomers (Fig. 1) belong to a family of chemicals named 2-arylpropionic acids, or profens, an important sub-class of the frequently prescribed and used drugs called nonsteroidal antiinflammatory drugs (NSAIDs).

In the last years, preparative chiral chromatography has become a more and more important separation process for the purification of pharmaceuticals and other added-value products. One reason chromatography is preferred is that the process allows both high yields and purities of both enantiomers. On the other hand, this technique is applicable to a wide variety of racemic mixtures, since chromatographic stationary phases for enantiomer separation are now available.

Among the different analytical methods for chiral separation of profens, high-performance liquid chromatography (HPLC) using chiral stationary phases (CSPs) has been the

\footnotetext{
* Corresponding author. Tel.: +351 273303 090; fax: +351 273313051 . E-mail address: pais@ipb.pt (L.S. Pais).
}

most employed. Particularly, high chiral recognition is provided by using the phenylcarbamate derivatives of polysaccharides (cellulose and amylose-based) as CSPs. The amylose 3,5dimethylphenylcarbamate (e.g. Chiralpak AD) is the most used for the separation of profens racemates [1-3].

The optimization of chiral separations in these adsorbents is frequently a complex task that requires, at a preparative scale, a careful selection of its operating conditions. In the case of binary or multicomponent mixtures, an additional complexity results from the competition between the different components in the interaction with the stationary phase. Therefore, one of the first steps of the preliminary study of a chromatographic separation process is the determination of the equilibrium competitive adsorption isotherms of the two enantiomers that will contribute to explain the retention mechanism and allow the prediction of the production rate recoveries and separation costs. Additionally, solubility of the racemate, selectivity and retention times are separation parameters very sensitive to changes in mobile phase composition. In fact, the use of continuous separation processes, such as simulated moving bed (SMB) technology, has achieved high throughputs when high feed concentrations and short cycle times were applied [4]. 
<smiles>CC(C(=O)O)c1cccc(C(=O)c2ccccc2)c1</smiles>

Fig. 1. Chemical structure of ketoprofen enantiomers.

The aim of this work is the measurement of adsorption equilibrium data of ketoprofen enantiomers, using the adsorption-desorption method, under different mobile phase compositions. Additionally, solubility measurements of ketoprofen enantiomers and pulse and breakthrough experiments on the different mobile phases were also performed. Modeling of adsorption data and simulation of fixed-bed and SMB operation were carried out to justify the choice of the mobile phase composition for the preparative separation of ketoprofen enantiomers.

\section{Experimental}

\subsection{Equipment and materials}

All the analysis were performed on a Jasco HPLC system with an UV-1575 multiwavelength detector set at $260 \mathrm{~nm}$, equipped with a preparative cell $(1.0 \mathrm{~mm})$. A manual Rheodyne $7725(\mathrm{i})$ injection valve was used with three different loops: $20 \mu \mathrm{L}$, $100 \mu \mathrm{L}$ and $1 \mathrm{~mL}$.

Two chiral chromatographic columns were used with the same adsorbent material (Chiralpak AD, Daicel Chemical Industries Ltd., Japan) and the same dimensions ( $250 \mathrm{~mm} L \times 4,6 \mathrm{~mm}$ i.d.). These two columns have different particle size: one column, with a particle size of $10 \mu \mathrm{m}$, was used for analytical purposes (measurement of enantiomers concentrations); the other, with a particle size of $20 \mu \mathrm{m}$, was used in the preparative chromatographic experiments (adsorption-desorption steps, pulses with high concentrations and high injection volumes, and breakthrough experiments). It must be pointed out that a particle size of $20 \mu \mathrm{m}$ is normally used for preparative separations, including SMB operation.

All isotherm measurements were performed at a constant temperature of $20^{\circ} \mathrm{C}$. Methanol, ethanol and $n$-hexane (Fluka, Buchs, Switzerland) were all HPLC grade. Trifluoroacetic acid (TFA) was spectrophotometric grade, and racemic ketoprofen was of analytical grade, purchased from Merck (Madrid, Spain).

\subsection{Solubility measurements}

The solubility measurements were carried out by using a gravimetric method [5]. It consists in preparing saturated solutions of racemic ketoprofen in different solvents, which are placed in a thermostatic water bath at a constant temperature of $25^{\circ} \mathrm{C}$. After equilibration, a volume of $5 \mathrm{~cm}^{3}$ of the clear saturated solution is transferred to a previously weighed glass vial of mass $m_{\mathrm{V}}$. The mass of the vial plus the saturated solution, $m_{\mathrm{VS}}$, is then measured. After, the vial is placed in an oven at $30^{\circ} \mathrm{C}$ for solvent evaporation, until the remaining mass of the sample does not change with time. Then, the final mass of vial and residue, $m_{\mathrm{VR}}$, is measured, and solubility, expressed in mass (g) of solute per unit mass $(\mathrm{kg}$ ) of solvent (on a solute-free basis) can be determined by using the following equation:

$S=\frac{\left(m_{\mathrm{VR}}-m_{\mathrm{V}}\right)}{\left(m_{\mathrm{VS}}-m_{\mathrm{VR}}\right)} \times 10^{3}$

\subsection{Determination of competitive adsorption isotherms by the adsorption-desorption method}

The experimental determination of the competitive adsorption isotherms was carried out using the adsorption-desorption method. In this method, the preparative column is saturated with a large amount of feed solution with known concentration of the two enantiomers, $C_{i}^{\mathrm{F}}$, until equilibrium is achieved. The column is then completely regenerated with eluent. The eluted volume, resulting from this desorption step, is collected and analyzed, in order to measure each enantiomer concentration. The mass balance

$C_{i}^{\mathrm{d}} V^{\mathrm{d}}=\varepsilon V_{\mathrm{c}} C_{i}^{\mathrm{F}}+(1-\varepsilon) V_{\mathrm{c}} q_{i}^{*}$

will allow to evaluate the concentration of each component retained in the particle, $q_{i}^{*}$, in equilibrium with the feed concentration, $C_{i}^{\mathrm{F}}$. In fact, $q_{i}^{*}$ is an overall retained concentration, which includes both the adsorbed material and the material in the fluid inside pores. This is consistent with the simulation of the chromatographic process, considering a model based on homogeneous particles. In Eq. (2), $C_{i}^{d}$ is the concentration of each component in the eluted solution collected in the desorption step, $V^{\mathrm{d}}$ is the eluted volume, $V_{\mathrm{c}}$ is the column volume $\left(V_{\mathrm{c}}=4.15 \mathrm{~mL}\right.$ ), and $\varepsilon$ is the external bed porosity (considering a homogeneous particle model; $\varepsilon=0.4$ ). This procedure will allow the determination of a unique point of the adsorption isotherm for each component $\left(C_{i}^{\mathrm{F}}, q_{i}^{*}\right)$. The entire adsorption isotherm measurement will require a set of adsorption-desorption experiments, using different feed concentrations.

In this work, several adsorption isotherm measurements were carried out, using racemic ketoprofen solutions at different concentrations and using three different mobile phase compositions: a 20\% ethanol $/ 80 \% n$-hexane mixture and two pure eluents; ethanol and methanol. All eluents include $0.01 \%$ of an acidic modifier (trifluoroacetic acid, TFA). For example, $1000 \mathrm{~mL}$ of the $20 \%$ ethanol $/ 80 \% n$-hexane mixture is prepared adding $200 \mathrm{~mL}$ ethanol, $800 \mathrm{~mL} n$-hexane and $100 \mu \mathrm{L}$ of TFA. The concentration of each ketoprofen enantiomer in the feed (racemic) and eluted solutions was evaluated by HPLC, equipped with the analytical column described before.

\section{Modeling}

\subsection{Modeling of competitive adsorption isotherms}

After the experimental evaluation of the adsorption data, an isotherm model must be proposed in order to allow the simulation of the adsorption behavior and the overall chromatographic process. The binary Langmuir model is usually a common choice for this purpose (subscripts 1 and 2 represent the less and the more retained enantiomers, respectively): 
Table 1

Model equations for fixed-bed chromatography (breakthrough), using the linear driving force model

Mass balance equations

$\frac{\partial C_{i}}{\partial \theta}=\frac{1}{P e} \frac{\partial^{2} C_{i}}{\partial x^{2}}-\frac{\partial C_{i}}{\partial x}-\frac{1-\varepsilon}{\varepsilon} \operatorname{St}\left(q_{i}^{*}-q_{i}\right)$

$\frac{\partial q_{i}}{\partial \theta}=\operatorname{St}\left(q_{i}^{*}-q_{i}\right)$ with $i=1$ and 2 (component)

Equilibrium isotherms

$q_{i}^{*}=f_{i}\left(C_{1}, C_{2}\right)$

Initial conditions

$\theta=0, \quad \forall x, \quad C_{i}=q_{i}=0$

Boundary conditions

$x=0, C_{i}-\frac{1}{P e} \frac{\mathrm{d} C_{i}}{\mathrm{~d} x}=C_{i}^{\mathrm{F}}$ with $C_{i}^{\mathrm{F}}$ known feed concentration

$x=1, \frac{\mathrm{d} C_{i}}{\mathrm{~d} x}=0$

$x=z / L$, dimensionless axial coordinate; $\theta=t / \tau$, dimensionless time variable; $P e=u_{i} L_{\mathrm{c}} / D_{\mathrm{ax}}$, Peclet number; $\varepsilon$, external bed porosity; $k$, mass transfer coefficient $\left(\mathrm{s}^{-1}\right) ; \tau=L_{\mathrm{c}} / u_{i}$, holdup time (s); $S t=k \tau$, massic Stanton number.

Table 2

Solubility (S) of racemic ketoprofen in the three solvent compositions $\left(T=25^{\circ} \mathrm{C}\right)$, expressed in mass $(\mathrm{g})$ of solute per unit mass $(\mathrm{kg})$ of solvent (on a solute-free basis)

\begin{tabular}{lc}
\hline Solvent composition & Solubility $(S)$ \\
\hline $20 \%$ ethanol/80\% $n$-hexane & 101.3 \\
$100 \%$ ethanol & 836.9 \\
$100 \%$ methanol & 1463 \\
\hline
\end{tabular}

$q_{1}^{*}=\frac{Q b_{1} C_{1}}{1+b_{1} C_{1}+b_{2} C_{2}} ; q_{2}^{*}=\frac{Q b_{2} C_{2}}{1+b_{1} C_{1}+b_{2} C_{2}}$

However, this Langmuir model usually fails in the prediction of the chromatographic enantioseparation process. It is well known that, for the generality of chiral systems, the selectivity factor, $\alpha$, decreases with the increase of the chiral species concentrations, which is not assumed by the Langmuir model, where $\alpha$ is constant: $\alpha=\left(q_{2}^{*} / C_{2}\right) /\left(q_{1}^{*} / C_{1}\right)=b_{2} / b_{1}$. To over-

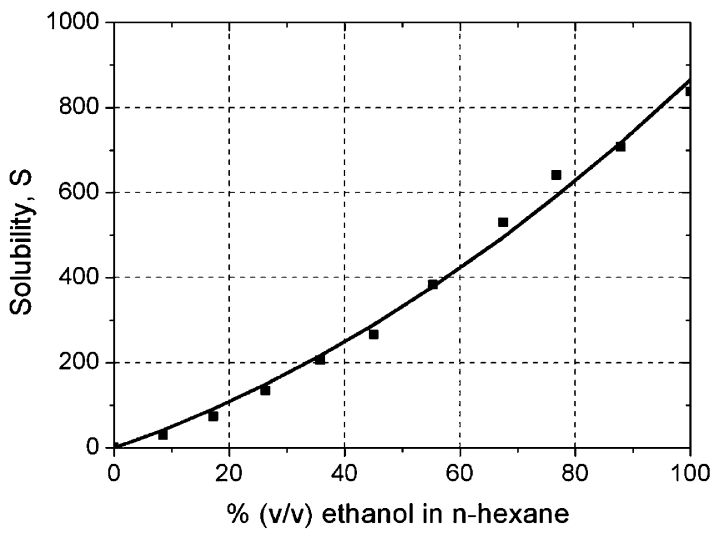

Fig. 2. Effect of the alcoholic content (ethanol $/ n$-hexane-based solvents) in the solubility (S) of racemic ketoprofen enantiomers at $25^{\circ} \mathrm{C}$, expressed in mass (g) of solute per unit mass $(\mathrm{kg})$ of solvent (on a solute-free basis).

come this limitation, more complex models are usually used, such as, the linear + Langmuir competitive isotherms (4) and (5) and the bi-Langmuir competitive model (6):

$$
\begin{aligned}
& q_{1}^{*}=m C_{1}+\frac{Q b_{1} C_{1}}{1+b_{1} C_{1}+b_{2} C_{2}} ; \\
& q_{2}^{*}=m C_{2}+\frac{Q b_{2} C_{2}}{1+b_{1} C_{1}+b_{2} C_{2}} \\
& q_{1}^{*}=m_{1} C_{1}+\frac{Q b_{1} C_{1}}{1+b_{1} C_{1}+b_{2} C_{2}} ; \\
& q_{2}^{*}=m_{2} C_{2}+\frac{Q b_{2} C_{2}}{1+b_{1} C_{1}+b_{2} C_{2}}
\end{aligned}
$$

$$
\begin{aligned}
& q_{1}^{*}=\frac{Q_{\mathrm{A}} b_{1} C_{1}}{1+b_{1} C_{1}+b_{2} C_{2}}+\frac{Q_{\mathrm{B}} b_{3} C_{1}}{1+b_{3} C_{1}+b_{4} C_{2}} ; \\
& q_{2}^{*}=\frac{Q_{\mathrm{A}} b_{2} C_{2}}{1+b_{1} C_{1}+b_{2} C_{2}}+\frac{Q_{\mathrm{B}} b_{4} C_{2}}{1+b_{3} C_{1}+b_{4} C_{2}}
\end{aligned}
$$

\begin{tabular}{|c|c|c|c|c|c|c|c|c|c|c|c|c|}
\hline Model & $M$ & $N$ & $m_{1}$ & $m_{2}$ & $Q_{\mathrm{A}}$ & $Q_{\mathrm{B}}$ & $b_{1}$ & $b_{2}$ & $b_{3}$ & $b_{4}$ & SQ & S.D. \\
\hline LG3 & \multirow{3}{*}{22} & 3 & - & - & \multicolumn{2}{|c|}{137.3} & $1.722 \times 10^{-2}$ & $1.875 \times 10^{-2}$ & - & - & 2.180 & 0.3388 \\
\hline LLG4 & & 4 & & & \multicolumn{2}{|c|}{52.19} & $3.586 \times 10^{-2}$ & $4.152 \times 10^{-2}$ & - & - & 1.493 & 0.2880 \\
\hline BLG6 & & 6 & - & - & 8.355 & 159.3 & $5.190 \times 10^{-2}$ & $1.479 \times 10^{-1}$ & $1.213 \times 10^{-2}$ & $1.149 \times 10^{-2}$ & 0.5920 & 0.1924 \\
\hline \multicolumn{13}{|c|}{$100 \%$ ethanol } \\
\hline LLG5 & \multirow[t]{2}{*}{22} & 5 & 0.5469 & 0.5931 & \multicolumn{2}{|c|}{24.75} & $1.986 \times 10^{-2}$ & $2.656 \times 10^{-2}$ & - & - & 0.1558 & 0.09573 \\
\hline BLG6 & & 6 & - & - & 0.5777 & 192.2 & $2.230 \times 10^{-1}$ & $6.595 \times 10^{-1}$ & $5.015 \times 10^{-3}$ & $5.771 \times 10^{-3}$ & 0.1291 & 0.08982 \\
\hline \multicolumn{13}{|c|}{$100 \%$ methanol } \\
\hline LG3 & \multirow[b]{3}{*}{22} & 3 & - & - & \multicolumn{2}{|c|}{177.9} & $5.707 \times 10^{-3}$ & $6.187 \times 10^{-3}$ & - & - & 0.4640 & 0.1563 \\
\hline LLG4 & & 4 & 1.366 & $10^{-5}$ & \multicolumn{2}{|c|}{177.2} & $5.734 \times 10^{-3}$ & $6.216 \times 10^{-3}$ & - & - & 0.4640 & 0.1606 \\
\hline LLG5 & & 5 & $7.024 \times 10^{-6}$ & $7.210 \times 10^{-8}$ & \multicolumn{2}{|c|}{177.2} & $5.734 \times 10^{-3}$ & $6.216 \times 10^{-3}$ & - & - & 0.4641 & 0.1652 \\
\hline
\end{tabular}

Table 3

Estimated model parameters for ketoprofen adsorption isotherms for the three mobile phase compositions

$M$ is the number of experimental points, $N$ the number of estimated parameters, $m_{1}, m_{2}, Q_{\mathrm{A}}, Q_{\mathrm{B}}, b_{1}, b_{2}, b_{3}$ and $b_{4}$ are the estimated parameters of the four isotherm models: LG3, Langmuir; LLG4, linear + Langmuir $\left(m_{1}=m_{2}\right)$; LLG5, linear + Langmuir $\left(m_{1} \neq m_{2}\right)$; BLG6, bi-Langmuir; SQ is the sum of squares of the residues and S.D. is the standard deviation. 
Contrary to the Langmuir isotherm (3), for these three models, selectivity is a concentration-dependent function.

\subsection{Breakthrough simulations}

In non-linear preparative chromatography, the modeling of band profiles can be done using the linear driving force model for fixed-bed chromatography. Table 1 presents the model equations for binary breakthrough experiments, including the mass balance equations, equilibrium isotherms, and initial and boundary
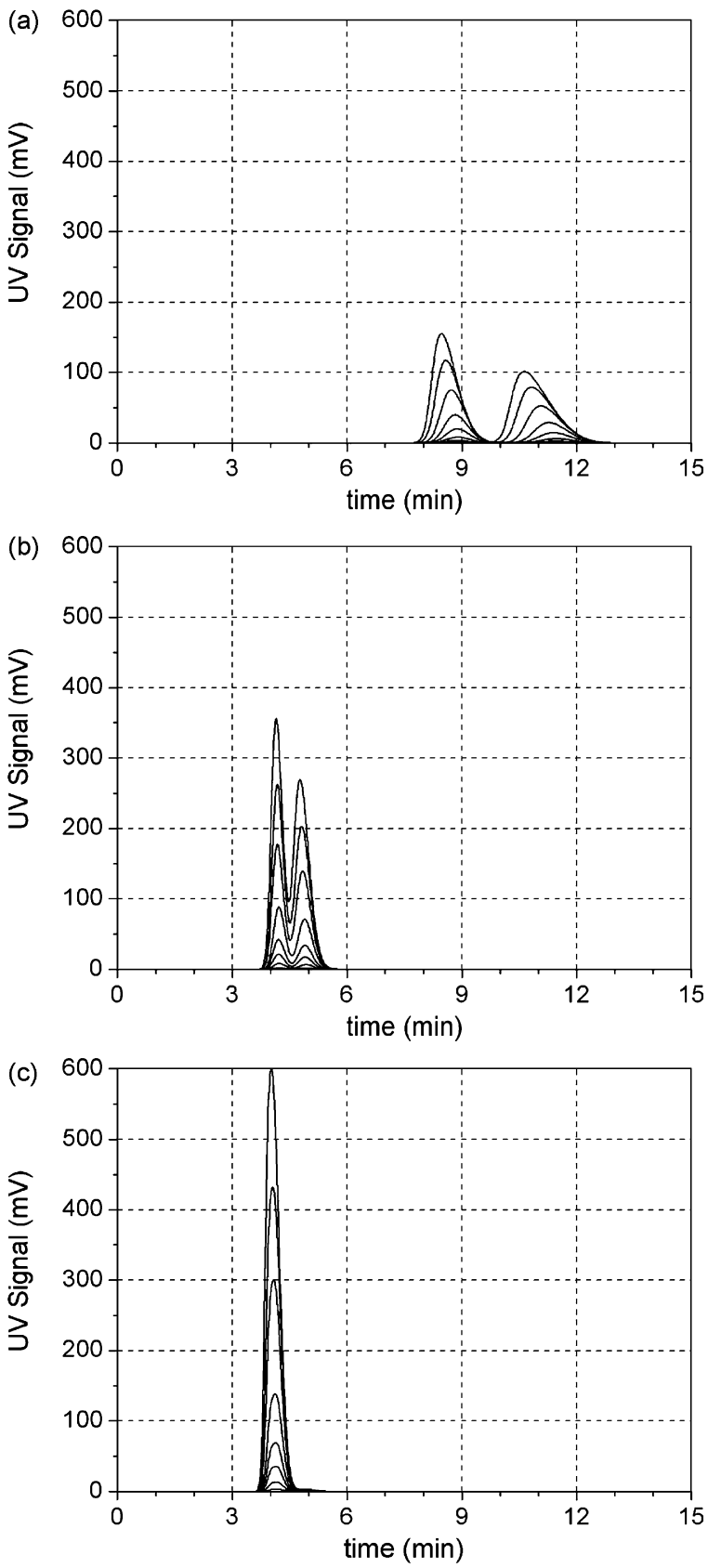

Fig. 3. Experimental elution profiles of ketoprofen enantiomers in the three different mobile phase compositions: (a) $20 \%$ ethanol $/ 80 \% n$-hexane; (b) $100 \%$ ethanol; (c) $100 \%$ methanol. Racemic ketoprofen concentrations in the range of $0.05-8.0 \mathrm{~g} / \mathrm{L}$; preparative column (particle diameter of $20 \mu \mathrm{m}$ ); UV detection at $260 \mathrm{~nm}$; flow rate of $1 \mathrm{~mL} / \mathrm{min}$; injected volume of $100 \mu \mathrm{L}$. conditions.

\section{Results and discussion}

\subsection{Solubility measurements}

The solubility of racemic ketoprofen mixtures was measured in the three different solvents: $100 \%$ methanol, $100 \%$ ethanol and $20 \%$ ethanol $/ 80 \% n$-hexane. Additionally, the dependence of ketoprofen solubility on the alcoholic content of an ethanol $/ n$ hexane-based solvent was also studied.
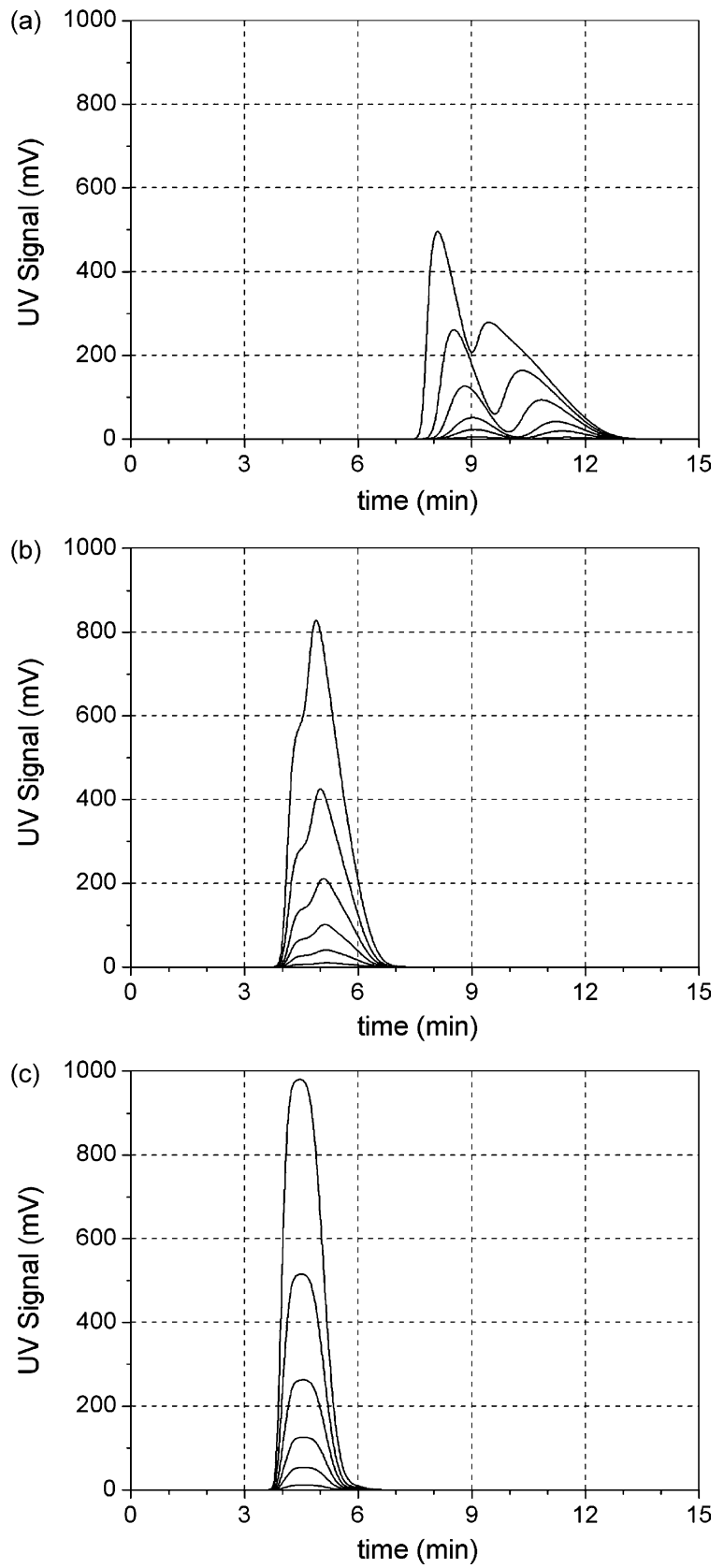

Fig. 4. Experimental elution profiles of ketoprofen enantiomers in the three different mobile phase compositions: (a) 20\% ethanol/80\% $n$-hexane; (b) $100 \%$ ethanol; (c) 100\% methanol. Racemic ketoprofen concentrations in the range of $0.05-4.0 \mathrm{~g} / \mathrm{L}$; preparative column (particle diameter of $20 \mu \mathrm{m}$ ); UV detection at $260 \mathrm{~nm}$; flow rate of $1 \mathrm{~mL} / \mathrm{min}$; injected volume of $1 \mathrm{~mL}$. 
Table 2 shows that ketoprofen enantiomers have increasing solubilities for $20 \%$ ethanol, pure ethanol and pure methanol. These results also confirm that racemic drugs have considerably higher solubilities in alcoholic solvents than in the traditional mobile phases used in analytical chiral separation, consisting in an alcohol-hydrocarbon combination, with high hydrocarbon
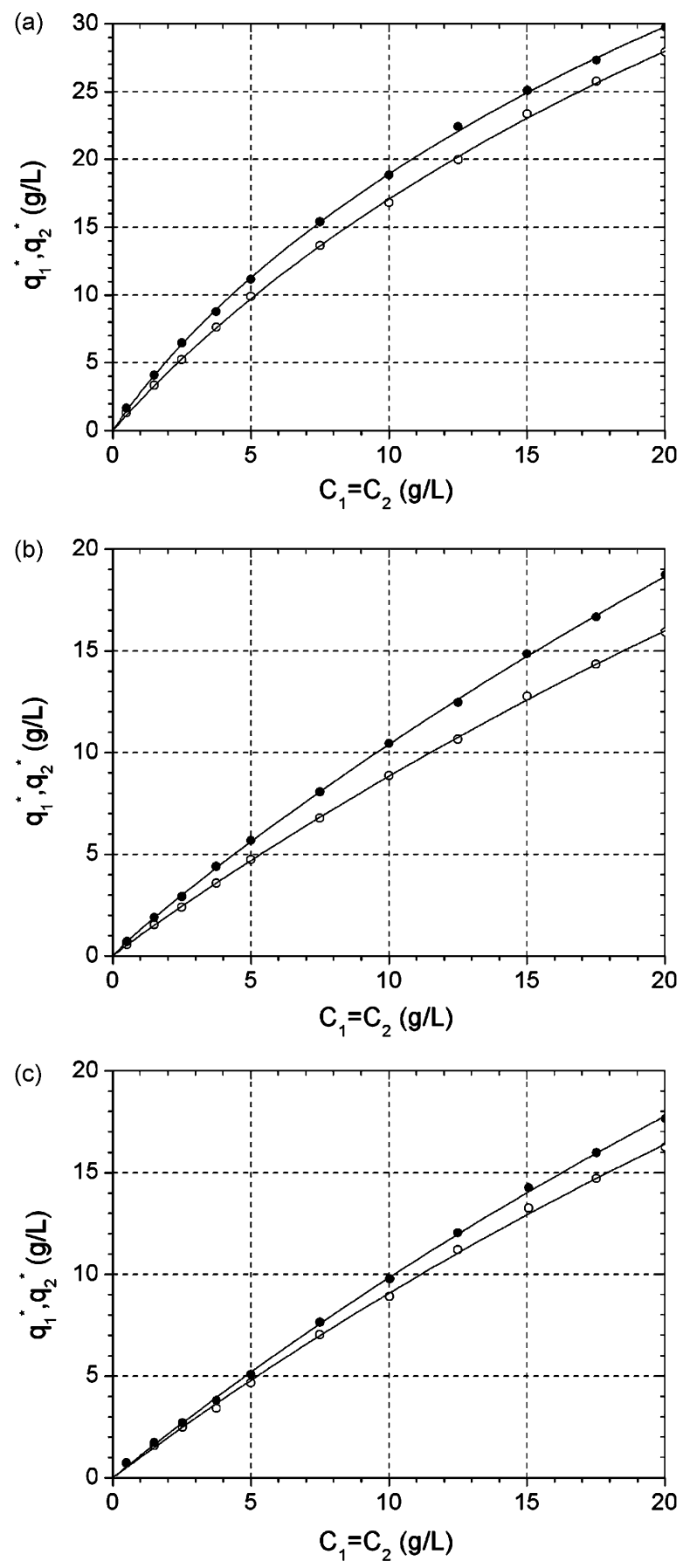

Fig. 5. Comparison between model and experimental results for the equilibrium adsorption isotherms: (a) 20\% ethanol/ $80 \% n$-hexane mobile phase, bi-Langmuir model (BLG6); (b) 100\% ethanol mobile phase, bi-Langmuir model (BLG6); (c) 100\% methanol mobile phase, Langmuir model (LG3). Open circles for experimental concentration of the less retained enantiomer; closed circles for experimental concentration of the more retained enantiomer; solid lines for adsorption isotherm model. content [6]. This conclusion is underlined with the experimental results obtained for the dependency of the racemic ketoprofen solubility on the alcoholic content of an ethanol $/ n$-hexanebased solvent, shown in Fig. 2. The ketoprofen enantiomers, which are insoluble in pure $n$-hexane, present increasing solubilities with the increase of the ethanol content. For pure ethanol, the solubility of racemic ketoprofen enantiomers is $836.9 \mathrm{~g} / \mathrm{kg}$ solvent. This result is in the same order of magnitude of the ones obtained by Gracin and Rasmunson for the solubility of ibuprofen enantiomers on pure methanol and ethanol [7].

These results clearly show the importance of using a mobile phase composition with a high alcoholic content, since, for preparative scale separations, the high solubility of the racemate is a major concern. The use of pure solvents will also be welcome at a preparative scale, because of the simplicity of their reutilization [8].

\subsection{Pulse experiments}

In order to have a global overview of the ketoprofen selectivity in the three different solvents and under preparative conditions (high concentrations), a set of preliminary pulse experiments was done. These experiments consisted of several pulse injections of different racemic ketoprofen concentrations in the preparative column (particle size of $20 \mu \mathrm{m}$ ) and using the $100 \mu \mathrm{L}$ and $1 \mathrm{~mL}$ injection loops. A flow rate of $1 \mathrm{~mL} / \mathrm{min}$ was used. Results obtained are shown in Fig. 3 (for $100 \mu \mathrm{L}$ loop) and Fig. 4 (for $1 \mathrm{~mL}$ loop). For all pulse experiments it can be seen that the increase of the amount injected leads to a decrease in the retention time of both enantiomers, which is a wellknown behavior for systems described by favorable isotherms. Figs. 3 and 4 show that the hydrocarbon mobile phase $(20 \%$ ethanol/80\% $n$-hexane) presents considerable higher retention times than the pure mobile phases (ethanol and methanol). The hydrocarbon mobile phase also leads to important chromatographic tails (see Fig. 4a), which is an indication of strong non-linear behavior and not welcome for preparative separations. Comparing the results obtained for the two pure alcohol

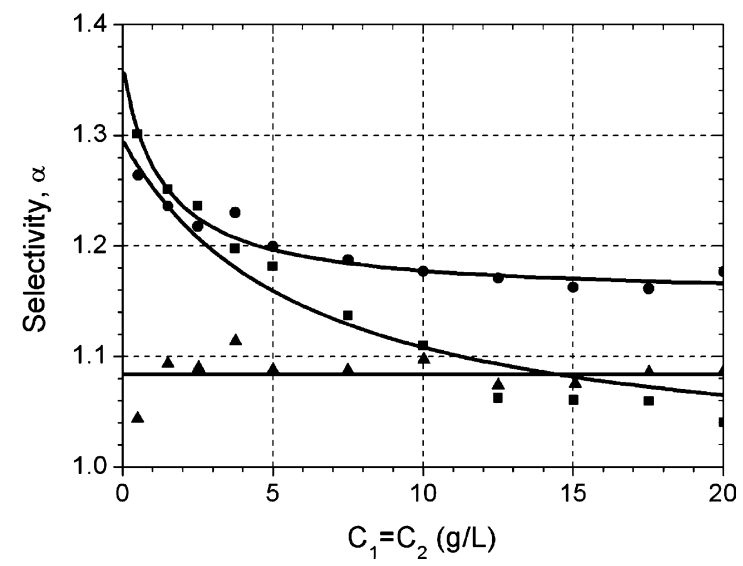

Fig. 6. Comparison between model (lines) and experimental (points) selectivities for racemic mixtures. Squares for $20 \%$ etanol $/ 80 \% n$-hexane, circles for $100 \%$ ethanol, and triangles for $100 \%$ methanol mobile phase. 

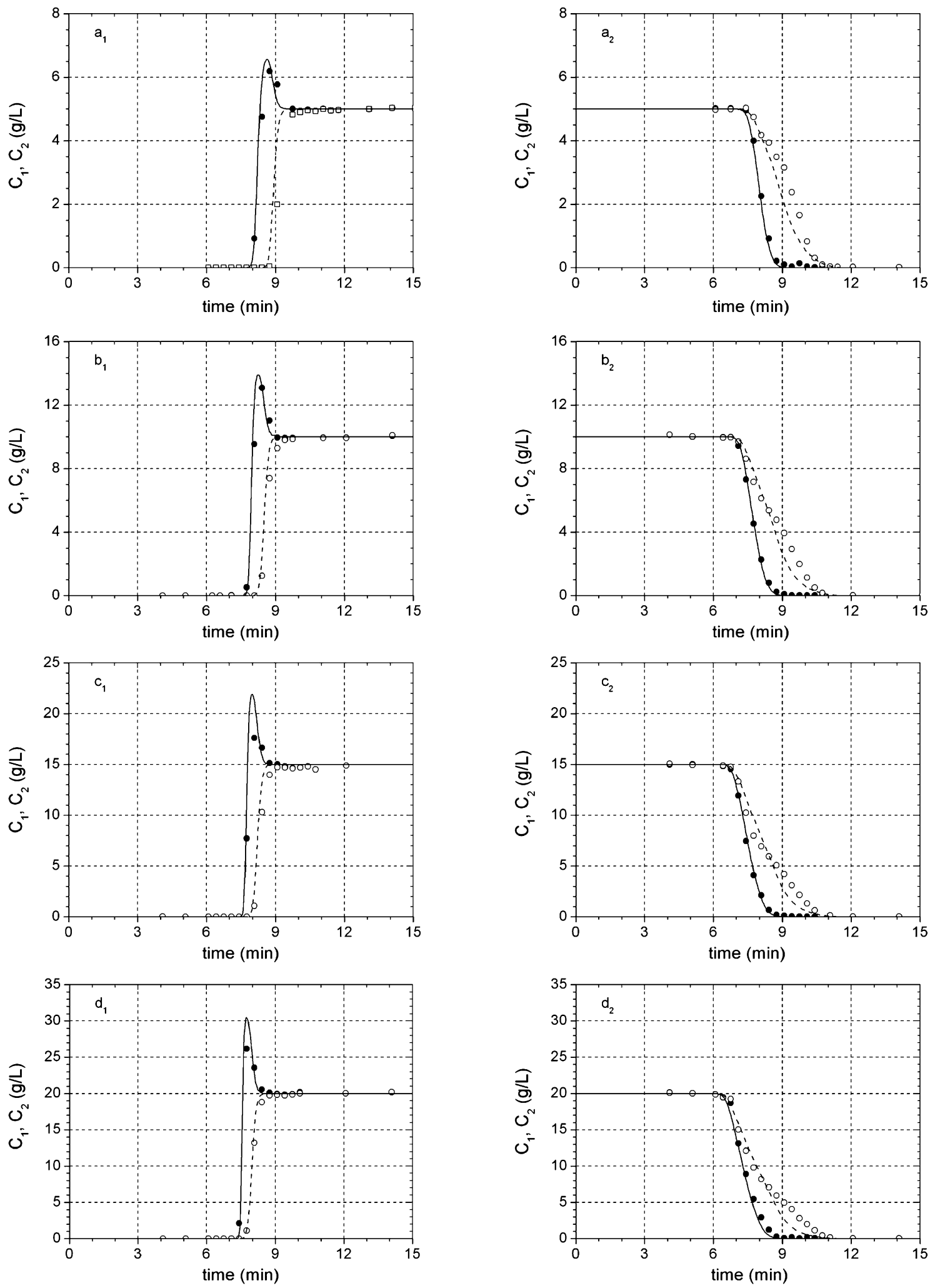

Fig. 7. Saturation (adsorption) and regeneration (desorption) curves for total feed concentrations of 10, 20, 30, and $40 \mathrm{~g} / \mathrm{L}$ (racemic mixtures). Comparison between experimental (points) and simulation (lines) results. Mobile phase: $100 \%$ ethanol; flow rate: $0.5 \mathrm{~mL} / \mathrm{min}$. Model parameters used: $\varepsilon=0.4, P e=3500, k=5.0 \mathrm{~s}^{-1}$ $(S t=k \tau=1000)$, and bi-Langmuir (BLG6) model parameters (see Table 3). 
mobile phases (Fig. 3b and c for $100 \mu \mathrm{L}$ and Fig. $4 \mathrm{~b}$ and c for $1 \mathrm{~mL}$ injection loops) it can be clearly concluded that, despite higher ketoprofen solubility, pure methanol does not allows acceptable selectivity values and, consequently, ketoprofen enantioseparation.

\subsection{Multicomponent adsorption isotherm experiments and modeling}

The experimental determination of competitive adsorption isotherms for ketoprofen enantiomers was carried out using the adsorption-desorption method for the three mobile phase compositions and fitted to the four isotherm models presented before (Eqs. (3)-(6)). The isotherm parameters were estimated using a Levenberg-Marquardt algorithm.

Table 3 presents the numerical results obtained and Fig. 5 shows the good agreement between model and experimental results for the equilibrium adsorption isotherms. For the $20 \%$ ethanol/80\% $n$-hexane and $100 \%$ ethanol mobile phases, although the linear +Langmuir model reasonably describes the adsorption behavior, more complex models, such as the bi-Langmuir isotherm, better simulate the experimental data obtained. For the $100 \%$ methanol mobile phase, the adsorption behavior is well described by the Langmuir model and no better results are obtained with more complex models (see Table 3 ).

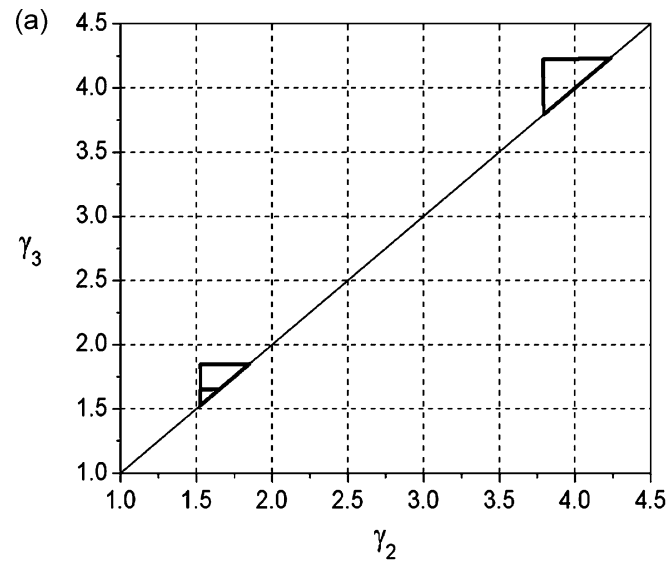

(c)

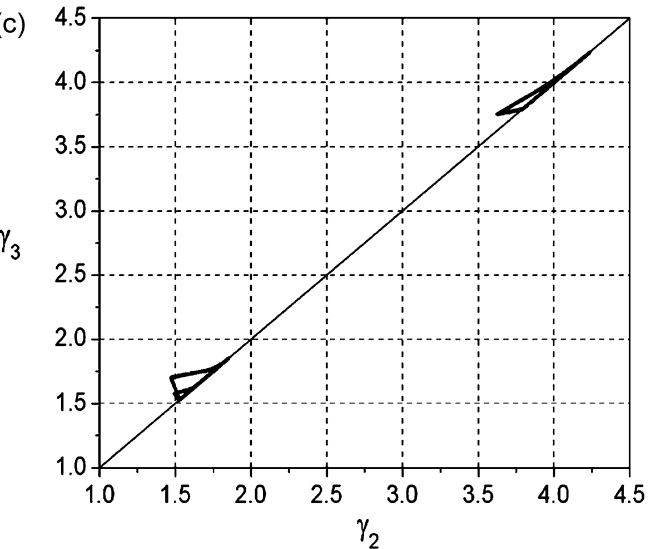

Fig. 6 compares the experimental and model selectivities for the three mobile phase compositions and illustrates three different scenarios. For $100 \%$ methanol, selectivity is low and constant, which means that the separation of ketoprofen enantiomers hardly can be achieved using pure methanol as mobile phase. The common $20 \%$ ethanol $/ 80 \% n$-hexane mobile phase, despite its high selectivity for low concentrations, presents a strong decrease in selectivity with the increase of enantiomers concentrations. The better situation is obtained for $100 \%$ ethanol, where selectivity maintains high values even for high enantiomer concentrations. In conclusion, pure ethanol can be used for ketoprofen enantioseparation, presenting better performances than the common $20 \%$ ethanol/ $80 \% n$-hexane mobile phase: it allows significantly higher enantiomer solubilities, lower retention times and significantly higher selectivities at high enantiomer concentrations. These are all aspects of utmost importance considering the preparative separation of ketoprofen enantiomers.

\subsection{Breakthrough experiments and simulation results}

In order to test the equilibrium adsorption isotherms measured, different saturation and regeneration curves were carried out for the pure ethanol mobile phase and for the whole range of feed concentrations (racemic mixtures of 10, 20,30, and

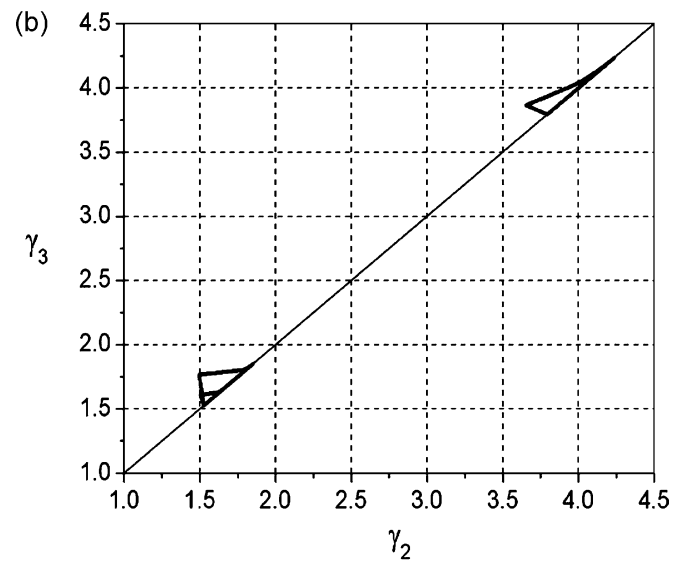

(d)

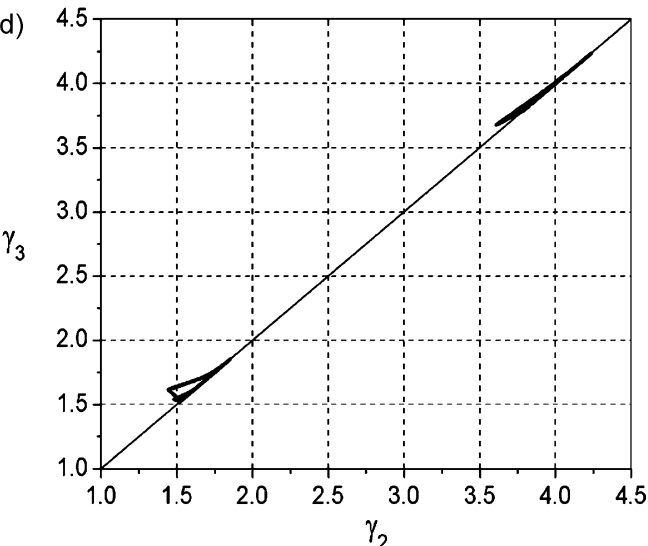

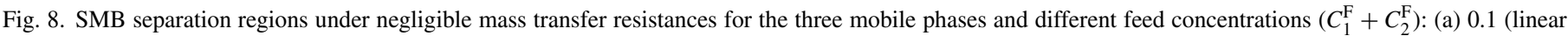

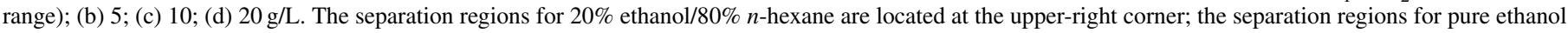
(bigger separation regions) and pure methanol (smaller separation regions) are located at the bottom-left corner. 
40 g/L). Fig. 7 shows the results obtained experimentally (by recovering and analyzing samples at different times) and predicted by the linear driving force model (Table 1). These figures show a very reasonable agreement between experimental and simulation results in the whole concentration range.

\subsection{Simulated moving bed simulations}

Chiral chromatographic separation processes are becoming of increasing importance in the development and the production of pharmaceutical drugs. Upon them, simulated moving bed (SMB) technology is being applied by an increasing number of pharmaceutical companies. Its use at a production scale is being considered as an alternative to the up to now leading techniques, such as enantioselective synthesis or diastereoisomeric crystallization.

It is interesting to predict and compare the performance of SMB operation for the separation of ketoprofen enantiomers using the three mobile phases in study. For this purpose, it was used the findings published by Morbidelli and co-workers, who developed a complete design of the binary countercurrent separation processes by SMB chromatography in the frame of equilibrium theory, assuming that mass transfer resistances and axial dispersion are negligible, and that the adsorption equilibria can be described through a variable selectivity modified Langmuir isotherm [9]. The SMB performance was evaluated for the three mobile phases by defining the complete separation regions and through the performance parameters of productivity and solvent consumption. A separation region is the area of possible SMB internal flow rates that allows $100 \%$ pure products (pure extract, only containing the more retained enantiomer, and pure raffinate, only containing the less retained species). The performance parameters of productivity and solvent consumption are evaluated at the vertex of each separation region. In fact, the vertex is the point at the boundary of the separation region most distant from the diagonal $\gamma_{3}=\gamma_{2}$ (see Fig. 8) and represents the

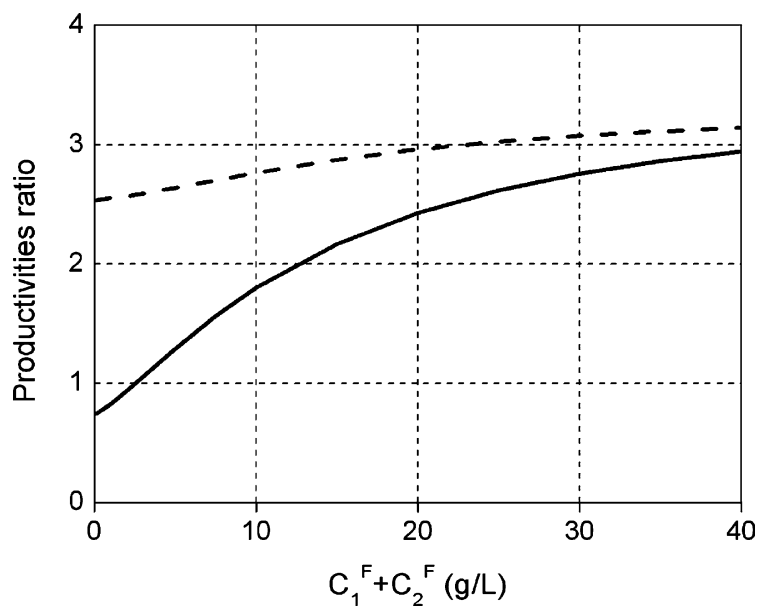

Fig. 9. Prediction of productivity of SMB operation under negligible mass transfer resistances as a function of feed concentration: ratio between the productivity obtained with $100 \%$ ethanol and the one obtained with $20 \%$ ethanol/80\% $n$ hexane (solid line) and ratio between the productivity obtained with $100 \%$ ethanol and the one obtained with $100 \%$ methanol (dashed line). best operating conditions in terms of system productivity and solvent consumption for a given feed concentration. For more information concerning SMB modeling and simulation, through the equilibrium theory and other more precise SMB models, see references [9-14].

Fig. 8 shows the separation regions obtained for the three mobile phases at different feed concentrations. The separation regions for $20 \%$ ethanol/80\% $n$-hexane (at the upper-right corner) have operating conditions considerable different from the ones obtained for the pure alcohol mobile phases (at the bottomleft corner), due to the higher retention times. This mobile phase also leads to a stronger dependency on feed concentration. Comparing the separation regions for the three mobile phases, it can be concluded that, for $20 \%$ ethanol $/ 80 \% n$-hexane, the separation region becomes quickly smaller with the increase of feed concentration. This is a sign of stronger non-linear behavior of the adsorption process and a reason for lower productivities. The comparison of the SMB performance for the two pure alcohol mobile phases is also clear: both have similar operating conditions due to similar retention times, but pure ethanol presents considerable better performances (bigger separation regions) due to higher selectivity, as shown previously in Figs. 5 and 6.

Figs. 9 and 10 stress out the conclusions taken from Fig. 8. Fig. 9 plots the ratios between the productivity obtained with pure ethanol and the ones obtained with the other two solvents, as a function of feed concentration. Fig. 10 plots the correspondent ratios of solvent consumptions. These simulation results also clearly show that pure ethanol is the better choice for the separation of ketoprofen enantiomers through SMB operation: at high feed concentrations (for example, $40 \mathrm{~g} / \mathrm{L}$ of racemic mixture; $20 \mathrm{~g} / \mathrm{L}$ of each enantiomer) the productivity using pure ethanol is three times the ones obtained with the other two solvents; the correspondent solvent consumption is only $75 \%$ and $25 \%$ of the one needed with pure methanol and $20 \%$ ethanol/80\% $n$-hexane, respectively. This last result also shows that, besides selectivity, retention times significantly influence solvent consumption in SMB operation.

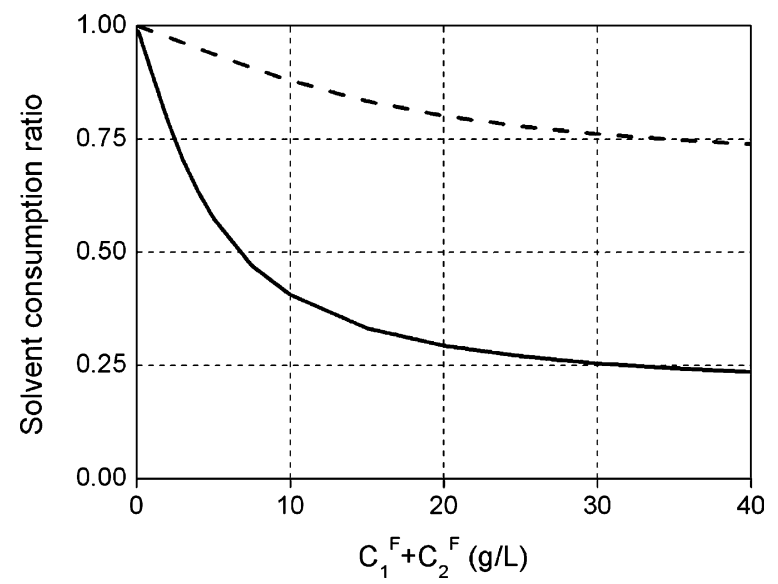

Fig. 10. Prediction of solvent consumption in SMB operation under negligible mass transfer resistances as a function of feed concentration: ratio between the solvent consumption with $100 \%$ ethanol and the one obtained with $20 \%$ ethanol/80\% $n$-hexane (solid line) and ratio between the solvent consumption with $100 \%$ ethanol and the one obtained with $100 \%$ methanol (dashed line). 


\section{Conclusions}

The optimization of preparative liquid chromatography and simulated moving bed for enantioseparation depends on the choice of the proper mobile phase. In this choice, a high resolution (or selectivity) of enantiomers should not be the only goal to be aimed. Other objectives, such as, to obtain high solubility of enantiomers and low retention times should also be taken into account.

This work shows that pure ethanol can be used for ketoprofen enantioseparation, instead of the mobile phase composed by $20 \%$ ethanol $/ 80 \% n$-hexane, which is the most commonly used in analytical chiral HPLC. The pure ethanol mobile phase allows, simultaneously, high enantiomers solubilities, low retention times and high selectivities at high feed concentrations. These are all aspects of utmost importance at a preparative and production scales, as it was shown for breakthrough adsorption processes and SMB operation.

\section{Acknowledgements}

Financial support by the Portuguese R\&D foundation FCT (Fundação para a Ciência e a Tecnologia) and European Community through FEDER (projects POCTI/EQU/38811/2001 and POCI/EQU/59738/2004), is gratefully acknowledged.

The authors wish to thank Simão P. Pinho (Bragança Polytechnic Institute) for the support on the solubility measurements.

\section{References}

[1] N.M. Maier, P. Franco, W. Lindner, Separation of enantiomers: needs, challenges, perspectives, J. Chromatogr. A 906 (2001) 3-33.
[2] E. Yashima, Polysaccharide-based chiral stationary phases for highperformance liquid chromatographic enantioseparation, J. Chromatogr. A 906 (2001) 105-125.

[3] K. Tachibana, A. Ohnishi, Reversed-phase liquid chromatography separation of enantiomers on polysaccharide type chiral stationary phases, J. Chromatogr. A 906 (2001) 127-154.

[4] E.R. Francotte, Enantioselective chromatography as a powerful alternative for the preparation of drug enantiomers, J. Chromatogr. A 906 (2001) 379-397.

[5] R.A. Granberg, A.C. Rasmunson, Solubility of paracetamol in pure solvents, J. Chem. Eng. Data 44 (1999) 1391-1395.

[6] L. Miller, C. Orihuela, R. Fronek, J. Murphy, Preparative chromatographic resolution of enantiomers using polar organic solvents with polysaccharide chiral stationary phases, J. Chromatogr. A 865 (1999) 211-226.

[7] S. Gracin, A.C. Rasmunson, Solubility of phenylacetic acid, phydroxyphenylacetic acid, $p$-aminophenylacetic acid, $p$-hydroxybenzoic acid, and ibuprofen in pure solvents, J. Chem. Eng. Data 47 (2002) 1379-1383.

[8] M. Schulte, R. Ditz, R. Devant, J. Kinkle, F. Charton, Comparison of the specific productivity of different chiral stationary phases used for simulated moving-bed chromatography, J. Chromatogr. A 769 (1997) 93-100.

[9] M. Mazzotti, G. Storti, M. Morbidelli, Optimal operation of simulated moving bed units for nonlinear chromatographic separations, J. Chromatogr. A 769 (1997) 3-24.

[10] L.S. Pais, J.M. Loureiro, A.E. Rodrigues, Modeling, simulation and operation of a simulated moving bed for continuous chromatographic separation of 1,1'-bi-2-naphthol enantiomers, J. Chromatogr. A 769 (1997) 25-35.

[11] L.S. Pais, J.M. Loureiro, A.E. Rodrigues, Separation of enantiomers of a chiral epoxide by simulated moving bed chromatography, J. Chromatogr. A 827 (1998) 215-233.

[12] C. Migliorini, A. Gentilini, M. Mazzotti, M. Morbidelli, Design of simulated moving bed units under nonideal conditions, Ind. Eng. Chem. Res. 38 (1999) 2400-2410.

[13] G. Biressi, O. Ludemann-Hombourger, M. Mazzotti, R.M. Nicoud, M. Morbidelli, Design and optimisation of a simulated moving bed unit: role of deviations from equilibrium theory, J. Chromatogr. A 876 (2000) 3-15.

[14] L.S. Pais, V.G. Mata, A.E. Rodrigues, Simulated moving bed and related techniques, in: G. Cox (Ed.), Preparative Enantioselective Chromatography, Blackwell Publishing, Oxford, UK, 2005, p. 176 (Chapter 7). 